\title{
Maternal microchimerism in cord blood and risk of childhood-onset type 1 diabetes
}

German Tapia ${ }^{1}$, Georgina Mortimer ${ }^{2}$, Jody $\mathrm{Ye}^{2}$, Benjamin Thomas Gillard ${ }^{2}$, Saranna Chipper-Keating ${ }^{2}$, Karl Mårild ${ }^{1}$, Marte K. Viken ${ }^{3}$, Benedicte A. Lie ${ }^{3,4}$, Geir Joner ${ }^{5,6}$, Torild Skrivarhaug $^{5,6}$, Pål R. Njølstad ${ }^{7,8}$, Ketil Størdal ${ }^{1,9}$, Kathleen M. Gillespie ${ }^{2}$, Lars C. Stene ${ }^{1}$

Affiliations:

${ }^{1}$ Norwegian Institute of Public Health, Oslo, Norway

${ }^{2}$ Diabetes and Metabolism, Bristol Medical School, University of Bristol, Bristol, UK

${ }^{3}$ Department of Immunology, Rikshospitalet, Oslo University Hospital, Oslo, Norway

${ }^{4}$ Department of Medical Genetics, University of Oslo, Oslo, Norway

${ }^{5}$ Division of Pediatric and Adolescent Medicine, Oslo University Hospital, Oslo, Norway

${ }^{6}$ Institute of Clinical Medicine, University of Oslo, Oslo, Norway

${ }^{7}$ Department of Pediatric and Adolescent Medicine, Haukeland University Hospital, Bergen, Norway

${ }^{8}$ KG Jebsen Center for Diabetes Research, Department of Clinical Science, University of Bergen, Bergen, Norway

${ }^{9}$ Pediatric Department, Østfold Hospital Trust, Grålum, Norway

Corresponding author: German Tapia, Department of Chronic Diseases and Ageing, Norwegian Institute of Public Health, P.O. Box 222 Skøyen, NO-0213 Oslo, Norway. E-mail: german.tapia@fhi.no

Running short title: Maternal microchimerism in cord blood and T1D

Word count: 1834 (abstract 245), 26 references.

Number of Figures, Tables: 1 Figure, 3 Tables.

This article has been accepted for publication and undergone full peer review but has not been through the copyediting, typesetting, pagination and proofreading process which may lead to differences between this version and the Version of Record. Please cite this article as doi: $10.1111 /$ pedi.12875 


\begin{abstract}
Background: Maternal microchimerism (MMc), the transmission of small quantities of maternal cells to the fetus, is relatively common and persistent. MMc has been detected with increased frequency in the circulation and pancreas of type 1 diabetes (T1D) patients. We investigated for the first time whether MMc levels at birth predict future T1D risk. We also tested whether cord blood MMc predicted MMc in samples taken at T1D diagnosis.
\end{abstract}

Methods: Participants in the Norwegian Mother and Child Cohort study were HLA class II typed to determine non-inherited, non-shared maternal alleles (NIMA). Droplet digital (dd) PCR assays specific for common HLA class II NIMA (HLADQB1*03:01, *04:02 and *06:02/03) were developed and validated. MMc was estimated as maternal DNA quantity in the fetal circulation, by NIMA specific ddPCR, measured in cord blood DNA from 71 children who later developed T1D and 126 controls within the cohort.

Results: We found detectable quantities of MMc in 34/71 future T1D cases (48\%) and 53/126 controls (42\%) (adjusted odds ratio (aOR) 1.27, 95\% Cl 0.68-2.36), and no significant difference in ranks of MMc quantities between cases and controls (Mann-Whitney $\mathrm{P}=0.46$ ). There was a possible association in the NIMA HLA$D Q B 1 * 03: 01$ subgroup with later T1D (aOR 3.89, 95\% Cl 1.05-14.4). MMc in cord blood was not significantly associated with MMc at T1D diagnosis.

Conclusions: Our findings did not support the hypothesis that the degree of MMc in cord blood predict T1D risk. The potential subgroup association with T1D risk should be replicated in a larger cohort.

Keywords: Microchimerism, HLA, Type 1 diabetes, Pregnancy, Childhood 


\section{Introduction}

Type 1 diabetes (T1D) is an autoimmune disease where insulin-producing beta cells become targeted by the immune system. The main known genetic risk factor for T1D is HLA genotype, and many weaker genetic risk factors exist ${ }^{1}$. Environmental factors are not well characterized, but it has been hypothesized that prenatal and early life factors influence T1D risk ${ }^{2,3}$.

Maternal transmission of cells to the fetus during pregnancy, known as maternal microchimerism (MMc), is believed to protect against fetal $\operatorname{loss}^{4}$, and promote regulatory $T$ cell mediated tolerance against non-inherited maternal antigens ${ }^{5}$. MMc has been shown to persist into adult life ${ }^{6}$, is relatively common ${ }^{7}$, and has been associated with both protective and adverse effects ${ }^{8,9}$. MMc has been postulated to play a role in autoimmune diseases, where it could be particularly relevant due to the strong association between certain HLA genotypes and autoimmune diseases ${ }^{8}$. MMc can be detected or quantified by targeting non-inherited, non-shared maternal allele (NIMA) DNA originating from maternal cells.

MMc has been detected in several tissues, amongst them islet beta cells ${ }^{10}$ in the pancreas, with increased frequency of MMc in T1D patients ${ }^{11}$. Higher levels of MMc in the circulation have also been shown for patients with T1D compared with healthy controls, using a quantitative PCR approach to detect non-inherited HLA alleles ${ }^{10}$. This increase could be due to MMc being present before development of T1D, or expansion of existing cells as a result of disease development. No studies to date however have investigated whether those who later develop disease have higher MMc levels prior to disease onset. Although the biological functions of MMc are largely unknown, we hypothesized that higher levels of pre-diagnostic MMc would contribute to lower risk of T1D.

Since MMc likely results from transplacental transfer via cord blood, we developed sensitive allele specific ddPCR assays to test for the first time whether cord blood 
MMc have any predictive value for childhood-onset T1D. Secondly, we investigated whether cord blood MMc predicted circulating MMc at diagnosis of T1D. 


\section{Research Design and Methods}

\section{Participants}

We designed a nested case-control study in the Norwegian Mother and Child Cohort Study $(\mathrm{MoBa})^{12}$, which recruited around 114,000 children and their mothers $(41 \%$ of eligible mothers participated) from all over Norway during 1999-2008. The current study uses data from repeated questionnaires, maternal postpartum and offspring cord blood samples ${ }^{13}$. All study participants gave written informed consent. The Norwegian Data Protection Authority and The Regional Committee for Medical Research Ethics approved MoBa and the current study. Characteristics of the participants are shown in Table 1. Baseline characteristics for those with available blood samples were largely similar to the whole MoBa cohort, except a lower proportion of caesarean section and premature birth (see ${ }^{14}$ ).

\section{Ascertainment of case status}

Children who developed T1D by February 5, 2014 were identified with a high degree of ascertainment by register linkage to the Norwegian Childhood Diabetes Registry ${ }^{15}$ and used as cases. A random set of controls was selected among participants in the MoBa cohort with available blood samples. We later ascertained if the initially selected random controls developed T1D until 1 June 2018. In total, 186 mother/child-pairs where the child developed T1D and 540 mother/child-pairs were used as controls. Of these, 71 T1D cases and 126 controls had an informative HLA for the NIMA ddPCR assays available: DQB1*03:01, *04:02, or *06:02/03 (Figure 1).

\section{Sampling}

Briefly, maternal venous blood samples were collected in EDTA tubes during pregnancy and shortly after birth, and a blood sample was taken at birth from the umbilical cord vein using a syringe $\mathrm{e}^{12,13,16}$. At diagnosis of T1D, venous blood samples were collected and shipped to the Norwegian Childhood Diabetes Registry. At the 
biobank repository, DNA was extracted and stored at $-20^{\circ} \mathrm{C}$ until analysis. For details of sample handling, see ${ }^{13,16,17}$.

\section{Genotyping}

To account for established T1D susceptibility markers, participants were genotyped using a custom Illumina Golden Gate assay (Illumina, San Diego, CA). DNA extraction, genotyping methods and quality control procedures are described in detail in a previous publication ${ }^{18}$. Briefly, tag-SNPs $(n=144)$ on chromosome 6 were used to impute human leukocyte antigen (HLA) class II genotype ${ }^{19}$, which was subsequently confirmed by classical HLA genotyping using allele specific PCR on all samples $^{20}$. For each informative dyad, we inferred the non-inherited non-shared HLADQB1 allele, NIMA (Figure 1, panel A).

\section{Droplet Digital (dd)PCR MMc quantification}

MMc were measured by quantifying the amount of NIMA specific DNA in cord blood. We developed allele-specific ddPCR assays for three common NIMAs (DQB1*03:01, DQB1*04:02, DQB1*06:02/*06:03), based on previous quantitative PCR assays ${ }^{10,21}$, to improve sensitivity for low copy number targets. A detailed protocol for the ddPCR method is available upon request. We used the droplet digital PCR QX200 system (Bio-Rad, California, USA). Final primer and probe concentrations optimized for DQB1*04:02 and DQB1*06:02/03 at 100nM and 300nM, and DQB1*03:01 at 300nM and 100nM respectively. DNA was quantified using the QuantiFluor dsDNA system (Promega, Madison, USA). Supplementary figure 1 shows the ddPCR profiles of a negative, genomic positive and positive cord blood sample for MMc DQB1*0602/3.

Sensitivity of assays determined using serial dilutions (500, 100, 5, 1 and 0.5 genomic equivalent ( $\mathrm{gEq})$ ) of NIMA positive homozygous DNA in a background DNA quantity of 10,000 genome equivalent negative for NIMA. Specificity for each assay was determined using well-characterized HLA cell lines. Ten wells were run for each sample, loaded at 10,000 gEq equal to 33ng; $100,000 \mathrm{gEq}$ was screened per 
sample. MMc value was determined by total copy number of HLA allele specific positive cells per 1000000 host cells as determined by beta-globin.

A priori, we decided to consider a sample positive only if two or more of the 10 replicates were positive. Samples with only one positive well $(n=62)$ were set to an MMc value of zero. Figure 1B shows the formation of the analysis sample.

As the measured MMc levels between probes are not directly comparable, we calculated MMc z-scores by subtracting the NIMA specific mean and dividing by the standard deviation (both calculated from control children), all done on log2transformed raw values.

\section{Other covariates}

A priori, we chose maternal pre-pregnancy body mass index (BMI) and maternal age as our primary adjusting variables, as these have been associated with $\mathrm{T}^{2} \mathrm{D}^{22-24}$ and could conceivably influence MMc. In a separate model we further included maternal smoking during pregnancy ${ }^{25}$ (coded as smoking or non-smoking at end of pregnancy), caesarean section ${ }^{26}$ and child`s HLA risk for T1D (coded as a binary variable; protective or neutral vs increased risk) as a sensitivity analysis. Distribution of these covariates are presented in Table 1.

Maternal age at delivery was obtained from the nationwide Medical Birth Registry of Norway (MBRN). Information regarding maternal pre-pregnancy BMI was obtained from mid-pregnancy questionnaires (accessible at www.fhi.no/moba) and MBRN.

\section{Statistical analysis}

We tested maternal microchimerism as a dichotomous variable (positive vs negative) and divided into three categories (negative, smedian of quantifiable MMc values, >median of the quantifiable MMc values), using logistic regression. We also tested whether the ranks of MMc differed between cases and controls using a MannWhitney $U$ test. In a secondary analysis, we stratified the above analysis by specific 
NIMA alleles. The statistical association between MMc in cord blood and at diagnosis of T1D in the same individual cases was estimated using logistic regression (for presence or absence of measurable MMc). To assess the sensitivity of our results towards the cut-off for MMc positivity, we reran the analysis when also calling samples positive if only one of 10 replicates were positive. All analyses were done in Stata Release 15 (College Station, Tx, USA). 


\section{Results}

Out of 726 genotyped mother-child dyads, 197 (71 T1D cases and 126 controls) provided data for the analysis (Figure 1). Of the 71 cases, a sample at the time of T1D diagnosis with valid MMc data was available from 60 . We measured a positive MMc value in 87/197 samples (44\%). The quantities measured in this study are shown Table 1.

\section{Differences between cases and controls}

Having any detectable MMc in cord blood was not significantly associated with later T1D (aOR 1.27, 95\% Cl 0.68-2.36, p=0.45). Investigating specific NIMAs, MMc tended to be associated with increased risk for later T1D in the group with HLADQB1*03:01 as the NIMA (Table 2). Investigating MMc quantity divided into three groups (no detectable NIMA vs. below or at median, and above median) gave similar results, with showed no significant associations with T1D overall, and a suggestive positive association for those with HLA-DQB1*03:01 as the NIMA (Table 2).

Comparing the ranks of the levels measured did not show any significant difference between cases and controls when summing all NIMAs (Mann-Whitney $P=0.46$ ), or in specific alleles (Mann-Whitney $P=0.07$ and 0.77 for HLA DQB1*03:01 and *06:02/03, respectively).

\section{Association between presence of MMc at birth and diagnosis}

There was a tendency for presence of MMc in cord blood to be associated with detectable MMc levels in the circulation at diagnosis of T1D (at an average of 6.1 years later), but this was not statistically significant (OR 2.21, 95\% Cl 0.76-6.46, $p=0.15)$.

\section{Sensitivity analyses}

Including more covariates, or changing the cut-off for positive MMc generally resulted in wider confidence intervals, but odds ratios were largely similar (Table 3). 


\section{Discussion}

In this first study of its kind, we tested whether cord blood MMc at birth was informative of future T1D risk, but we found no significant overall association.

An important strength of this study was the use of digital droplet PCR, which allowed us to investigate the presence of MMc in a more sensitive and specific manner than earlier studies. We observed more children with detectable MMc than a previous study from Korea ( $44 \%$ in our study vs $23 \%$ ), but we use ddPCR which is considered a more sensitive method. Earlier studies in T1D have investigated individuals after diagnosis in peripheral blood and pancreatic tissue ${ }^{10,11}$. Our results are not consistent with results from these, which could be due to MMc differences between cord blood at birth, and in the pancreas or circulation, as we found no increased risk associated with the presence, or levels, of total MMc. We found a possible association in the HLA-DQB1*03:01 NIMA subgroup with later T1D, which must be interpreted with caution.

While maternal cells are believed to cross into the fetus through the umbilical cord, with increased frequency from mid pregnancy towards term, it is only feasible to measure MMc levels on the day of delivery. In theory, transfer of cells could fluctuate temporally. Our study does not rule out an important role of MMc in T1D, for example in terms of specific cell types or their functions in specific tissues postnatally ${ }^{10,11}$. This is an observational study, and we cannot rule out unknown factors influencing MMc levels and later disease. Although the current analysis emanate from a large undertaking, the number of children with informative and measurable MMc in the final analysis was somewhat limited. Because of the strong protective effect of DQB1*06:02, this allele is known to be relatively over-represented as the NIMA in T1D cases but the data presented here suggest that this allele does not confer any additional protective effect through MMc. 
In conclusion, our findings do not support a protective role of pre-diagnostic MMc in T1D, as maternal microchimerism measured in cord blood did not significantly predict the risk of future T1D in children. 


\section{Conflicts of interest}

No other potential conflict of interest relevant to this article was reported. The authors alone are responsible for the content and writing of the paper.

\section{Acknowledgements}

The authors are grateful to Professor Klaus Badenhoop, Goethe University Frankfurt, Germany, for providing samples used to develop the ddPCR method. The Norwegian Mother and Child Cohort Study are supported by the Norwegian Ministry of Health and Care Services and the Ministry of Education and Research, NIH/NIEHS (contract no N01-ES-75558), NIH/NINDS (grant no.1 UO1 NS 047537-01 and grant no.2 UO1 NS 047537-06A1). We are grateful to all the participating families in Norway who take part in this on-going cohort study. The Norwegian Childhood Diabetes Registry is funded by The South-Eastern Norway Regional Health Authority. We are thankful to the Norwegian Childhood Diabetes Registry and to the Norwegian Childhood Diabetes Study Group. Dr. Størdal was supported by an unrestricted grant from the Oak Foundation, Geneva, Switzerland. Costs of all data acquisition, including laboratory assays in MoBa (the sub-study PAGE; Prediction of Autoimmune Diabetes and Celiac Disease in Childhood by Genes and Perinatal Environment), was supported by grant 2210909/F20 from the Norwegian Research Council (Dr. Stene). Dr. Njølstad was supported by the European Research Council (AdG \#293574), Stiftelsen Kristian Gerhard Jebsen, the Research Council of Norway (\#240413), the Bergen Research Foundation, and the Western Health Authorities (Strategic Grant). Data from the Norwegian Patient Register has been used in this publication. The interpretation and reporting of these data are the sole responsibility of the authors, and no endorsement by the Norwegian patient register is intended nor should be inferred. SNP genotyping in this study was performed at the Genomics Core Facility, Norwegian Radium hospital, Oslo, Norway.

\section{Author contributions:}

Conception and design: LCS, GT, KS, KMG 
Acquisition of pregnancy cohort data: LCS, KS.

Acquisition of childhood incident T1D data: TS, GJ, PRN.

SNP Genotyping and HLA imputation: GT, MKV, BAL.

HLA typing: GM, JY, BG, SCK, KMG.

Development of ddPCR method and experimental procedures: GM, JY, BG, SCK, KMG.

Data cleaning and preparation: GT

Planning statistical analyses: LCS, GT, KS.

Performing statistical analyses: GT.

Interpretation of data: All authors.

Drafting the manuscript: GT

Revising the manuscript critically for important intellectual content: All authors.

Final approval of the version to be published: All authors

Taking responsibility for the integrity of the data and the accuracy of the data analysis: GT, LCS, KMG.

Obtaining funding: LCS, KS, KMG 


\section{References}

1. Pociot F, Lernmark Å. Genetic risk factors for type 1 diabetes. Lancet. 2016;387(10035):23312339.

2. Rewers M, Ludvigsson J. Environmental risk factors for type 1 diabetes. Lancet. 2016;387(10035):2340-2348.

3. Stene LC, Gale EA. The prenatal environment and type 1 diabetes. Diabetologia. 2013;56(9):1888-1897.

4. Kinder JM, Jiang TT, Ertelt JM, et al. Cross-Generational Reproductive Fitness Enforced by Microchimeric Maternal Cells. Cell. 2015;162(3):505-515.

5. Mold JE, Michaelsson J, Burt TD, et al. Maternal alloantigens promote the development of tolerogenic fetal regulatory T cells in utero. Science. 2008;322(5907):1562-1565.

6. Maloney S, Smith A, Furst DE, et al. Microchimerism of maternal origin persists into adult life. J Clin Invest. 1999;104(1):41-47.

7. Roh EY, Yoon JH, Shin S, Song EY, Chung HY, Park MH. Frequency of fetal-maternal microchimerism: an analysis of the HLA-DRB1 gene in cord blood and maternal sample pairs. J Matern Fetal Neonatal Med. 2017;30(21):2613-2619.

8. Nelson JL. The otherness of self: microchimerism in health and disease. Trends Immunol. 2012;33(8):421-427.

9. Kinder JM, Stelzer IA, Arck PC, Way SS. Immunological implications of pregnancy-induced microchimerism. Nat Rev Immunol. 2017;17(8):483-494.

10. Nelson JL, Gillespie KM, Lambert NC, et al. Maternal microchimerism in peripheral blood in type 1 diabetes and pancreatic islet beta cell microchimerism. Proc Natl Acad Sci U S A. 2007;104(5):1637-1642.

11. Ye J, Vives-Pi M, Gillespie KM. Maternal microchimerism: increased in the insulin positive compartment of type 1 diabetes pancreas but not in infiltrating immune cells or replicating islet cells. PLoS One. 2014;9(1):e86985.

12. Magnus P, Birke C, Vejrup K, et al. Cohort Profile Update: The Norwegian Mother and Child Cohort Study (MoBa). Int J Epidemiol. 2016;45(2):382-388.

13. Rønningen KS, Paltiel L, Meltzer HM, et al. The biobank of the Norwegian Mother and Child Cohort Study: a resource for the next 100 years. Eur J Epidemiol. 2006;21(8):619-625.

14. Stordal K, Marild K, Tapia G, et al. Fetal and maternal genetic variants influencing neonatal vitamin D status. J Clin Endocrinol Metab. 2017.

15. Skrivarhaug T, Stene LC, Drivvoll AK, Strøm H, Joner G, Norwegian Childhood Diabetes Study G. Incidence of type 1 diabetes in Norway among children aged 0-14 years between 1989 and 2012: has the incidence stopped rising? Results from the Norwegian Childhood Diabetes Registry. Diabetologia. 2014;57(1):57-62.

16. Paltiel L, Haugan A, Skjerden T, et al. The biobank of the Norwegian Mother and Child Cohort Study - present status. Nor J Epidemiol. 2014;24(1-2):29-35.

17. Vistnes $\mathrm{M}$, Tapia $\mathrm{G}$, Mårild $\mathrm{K}$, et al. Plasma immunological markers in pregnancy and cord blood: A possible link between macrophage chemo-attractants and risk of childhood type 1 diabetes. Am J Reprod Immunol. 2018;79(3).

18. Mårild K, Vistnes M, Tapia G, et al. Midpregnancy and cord blood immunologic biomarkers, HLA genotype, and pediatric celiac disease. J Allergy Clin Immunol. 2017;139(5):1696-1698.

19. Dilthey A, Leslie S, Moutsianas L, et al. Multi-population classical HLA type imputation. PLoS Comput Biol. 2013;9(2):e1002877. 
20. Aitken RJ, Mortimer GL, Gillespie KM. Type 1 Diabetes High-Risk HLA Class II Determination by Polymerase Chain Reaction Sequence-Specific Primers. Methods Mol Biol. 2016;1433:13-

20.

21. Lambert NC, Erickson TD, Yan Z, et al. Quantification of maternal microchimerism by HLAspecific real-time polymerase chain reaction: studies of healthy women and women with scleroderma. Arthritis Rheum. 2004;50(3):906-914.

22. Cardwell CR, Stene LC, Joner G, et al. Maternal age at birth and childhood type 1 diabetes: a pooled analysis of 30 observational studies. Diabetes. 2010;59(2):486-494.

23. Stene LC, Magnus P, Lie RT, Søvik O, Joner G. Maternal and paternal age at delivery, birth order, and risk of childhood onset type 1 diabetes: population based cohort study. BMJ. 2001;323(7309):369.

24. Magnus MC, Olsen SF, Granstrom C, et al. Paternal and maternal obesity but not gestational weight gain is associated with type 1 diabetes. Int J Epidemiol. 2018;47(2):417-426.

25. Magnus MC, Tapia G, Olsen SF, et al. Parental Smoking and Risk of Childhood-onset Type 1 Diabetes. Epidemiology. 2018;29(6):848-856.

26. Cardwell CR, Stene LC, Joner G, et al. Caesarean section is associated with an increased risk of childhood-onset type 1 diabetes mellitus: a meta-analysis of observational studies.

Diabetologia. 2008;51(5):726-735. 
Table 1: Characteristics of cases with childhood type 1 diabetes and randomly selected controls in the present study.

Cases $(\mathrm{n}=\mathbf{7 1})$ $11.5(7.3-15.1)$

Median age (range) at end of follow-up* (years)

Female sex

Preterm birth

$\mathrm{Pa}$ ity

.vo earlier births

e

Two or more

- ternal age (years (median, range))

1024

25-34

$\checkmark-42$

Maternal smoking during pregnancy

ivun-smoker at end of pregnancy ${ }^{\ddagger}$

Sr oked at end of pregnancy

Missing data

Pre-pregnancy BMI (kg/m² (median, range))

$<25$

25-30

$>30$

.vilssing data

Child's HLA genotype ${ }^{\S}$

...otective (DQ6)

$N \wedge$ tral (any other HLA not mentioned)

Increased risk ( $\geq 1$ copy of either DQ8 or DQ2.5)

High risk (DQ8/DQ2.5 heterozygote)

cuucurean section"

MMc quantities (median (range)")

B1*03:01 NIMA in cord blood

DCB1*03:01 NIMA at diagnosis

B $1^{*} 04: 02$ NIMA in cord blood

DCB1*04:02 NIMA at diagnosis

ก $B 1{ }^{*} 06: 02 / 3$ NIMA in cord blood

DOB1*06:02/3 NIMA at diagnosis
$34(47.9 \%)$
$5(7.0 \%)$

$35(49.3 \%)$

$20(28.2 \%)$

$16(22.5 \%)$

$30(22-42)$

$4(5.6 \%)$

$55(77.5 \%)$

$12(16.9 \%)$

$61(85.9 \%)$

$7(9.9 \%)$

$3(4.2 \%)$

$26.1(17.3-38.6)$

$28(39.4 \%)$

$27(38.0 \%)$

$10(14.1 \%)$

$6(8.5 \%)$

$2(2.8 \%)$

$3(4.2 \%)$

$38(53.5 \%)$

$28(39.4 \%)$

$8(10.8 \%)$

$55.4(10.9-867.7)$

$43.5(10.0-221.2)$

$65.0(16.8-76.6)$

$50.3(16.8-59.2)$

$30.9(15.2-343.8)$

$55.8(13.2-190.4)$
Controls $(n=126)$

$10.5(7.0-15.9)$

64 (50.8\%)

$4(3.2 \%)$

60 (47.6\%)

$40(31.7 \%)$

$26(20.6 \%)$

$30(19-40)$

17 (13.5\%)

$86(68.3 \%)$

$23(18.3 \%)$

$104(82.5 \%)$

17 (13.5\%)

$5(4.0 \%)$

$24.1(18.7-37.8)$

67 (53.2\%)

$34(27.0 \%)$

$12(9.5 \%)$

$13(10.3 \%)$

$21(16.7 \%)$

$29(23.0 \%)$

68 (54.0\%)

$8(6.3 \%)$

$15(11.2 \%)$

$46.8(16.7-588.8)$

$17.2(16.2-144.8)$

$48.6(9.5-1010.2)$ 
* The diagnosis date of the last case included - June 2, 2016

$\dagger$ The median age at diagnosis of T1D cases was 6.3 (range $0.7-13.0$ ) years

¥ Including those that quit smoking shortly before or during pregnancy, as the association with T1D has been observed in those that smoked throughout pregnancy ${ }^{25}$.

$\S$ Coded as a binary variable in the analysis - protective or neutral $(0)$ vs increased or high risk (1). The increased risk category ( $\geq 1$ copy of either DQ8 [DQA1*03-DQB1*03:02-

DRB1*04] or DQ2.5 [DQA1*05:01-DQB1*02:01] also required no HLA DQ6 [DQA1*01:02DQB1*06:02-DRB1*15:01] protective allele).

II Includes unknown $(n=1)$, emergency $(n=9)$ and elective $(n=13)$ caesarean section Tा Median and range of positive values. 
Table 2: Association between MMc in cord blood and type 1 diabetes

\begin{tabular}{|c|c|c|c|c|c|}
\hline & Cases $^{\dagger}$ & Controls $^{\dagger}$ & OR $(95 \% \mathrm{Cl})$ & aOR $(95 \% \mathrm{Cl})^{\star}$ & $\begin{array}{r}\text { Adjusted } \\
\text { P-value }\end{array}$ \\
\hline \multicolumn{6}{|l|}{ Presence of detectable MMc } \\
\hline Any $^{\ddagger}$ NIMA & $32 / 65$ & $49 / 113$ & $1.27(0.71-2.27)$ & $1.27(0.68-2.36)$ & 0.45 \\
\hline DQB1*03:01 NIMA & $14 / 20$ & $16 / 36$ & $2.44(0.81-7.35)$ & $3.89(1.05-14.44)$ & 0.04 \\
\hline —QB $1 * 06: 02 / 03$ NIMA & $15 / 33$ & $64 / 64$ & $1.04(0.47-2.31)$ & $0.96(0.40-2.27)$ & 0.92 \\
\hline \multicolumn{6}{|l|}{ Categorical analysis ${ }^{\S}$} \\
\hline Any ${ }^{+}$NIMA, Undetected & $33 / 65$ & $64 / 113$ & Ref. & Ref. & \\
\hline $\mathrm{Any}^{\ddagger} \mathrm{N}^{\prime} \mathrm{A} \mathrm{A} \leq$ median & $18 / 65$ & $26 / 113$ & $1.52(0.75-3.07)$ & $1.34(0.64-2.81)$ & 0.44 \\
\hline Any $^{\mp}$ NIMA $>$ median & $14 / 65$ & $23 / 113$ & $1.02(0.48-2.18)$ & $1.20(0.54-2.65)$ & 0.66 \\
\hline Per category increase & $65 / 65$ & $113 / 113$ & $1.07(0.74-1.53)$ & $1.12(0.76-1.65)$ & 0.56 \\
\hline 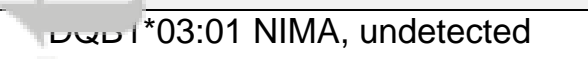 & $6 / 20$ & $20 / 36$ & Ref. & Ref. & \\
\hline DQB1*03:01 NIMA $\leq$ median & $6 / 20$ & $9 / 36$ & $2.10(0.55-7.99)$ & $2.74(0.61-12.44)$ & 0.19 \\
\hline DQB1*03:01 NIMA > median & $8 / 20$ & $7 / 36$ & $2.79(0.78-10.01)$ & $5.77(1.21-27.52)$ & 0.03 \\
\hline D ategory increase & $20 / 20$ & $36 / 36$ & $1.68(0.89-3.17)$ & $2.40(1.10-5.24)$ & 0.03 \\
\hline DQB1*06:02/03 NIMA, undetected & $18 / 33$ & $35 / 64$ & Ref. & Ref. & \\
\hline DQB1*06:02/03 NIMA $\leq$ median & $11 / 33$ & $15 / 64$ & $1.65(0.66-4.11)$ & $1.36(0.52-3.62)$ & 0.54 \\
\hline DOB1*06:02/03 NIMA > median & $4 / 33$ & $14 / 64$ & $0.48(0.14-1.61)$ & $0.51(0.14-1.83)$ & 0.30 \\
\hline Per category increase & $33 / 33$ & $64 / 64$ & $0.82(0.49-1.36)$ & $0.80(0.45-1.40)$ & 0.43 \\
\hline
\end{tabular}

OR: Odds Ratio; aOR: adjusted OR; Cl: Confidence Interval

* adjusted for maternal age and pre-pregnancy BMI

$\dagger$ included in the adjusted analysis. There were 71 cases and 126 controls in total in the unadjusted analyses.

‡ combining DQB1*03:01, *04:02 and *06:02/03. The DQB1*04:02 NIMA subgroup was not analyzed separately due to low number of children with measurable quantities in this group.

$\S$ the median value was calculated from controls with detectable levels 
Table 3: Sensitivity analyses

\begin{tabular}{|c|c|c|c|}
\hline & $\begin{array}{l}\text { aOR }(95 \% \mathrm{Cl})^{\star} \\
\text { Main analysis }\end{array}$ & $\begin{array}{c}\text { aOR }(95 \% \mathrm{Cl})^{\dagger}, \\
\text { additionally adjusted }\end{array}$ & $\begin{array}{c}\text { aOR }(95 \% \mathrm{Cl})^{\star} \text {, including } \\
\text { unreplicated positives }\end{array}$ \\
\hline \multicolumn{4}{|l|}{ Presence of detectable MMc } \\
\hline Any $^{\ddagger}$ NIMA & $1.27(0.68-2.36)$ & $1.28(0.65-2.52)$ & $1.19(0.62-2.27)$ \\
\hline DQB1*03:01 NIMA & $3.89(1.05-14.44)$ & $6.33(0.79-50.75)$ & $2.39(0.56-10.26)$ \\
\hline DQB1*06:02/03 NIMA & $0.96(0.40-2.27)$ & $1.05(0.42-2.65)$ & $1.26(0.49-3.20)$ \\
\hline \multicolumn{4}{|l|}{ Categorical analysis ${ }^{\S}$} \\
\hline Any NIMA, Undetected & Ref. & Ref. & Ref. \\
\hline Anv $\mathrm{NIMA} \leq$ median & $1.34(0.64-2.81)$ & $1.42(0.63-3.20)$ & $0.75(0.33-1.69)$ \\
\hline Anv $^{\ddagger}$ NIMA $>$ median & $1.20(0.54-2.65)$ & $1.12(0.47-2.69)$ & $1.35(0.80-3.40)$ \\
\hline Per category increase & $1.12(0.76-1.65)$ & $1.10(0.72-1.68)$ & $1.29(0.89-1.87)$ \\
\hline UQB1*03:01 NIMA, undetected & Ref. & Ref. & Ref. \\
\hline DQB1*03:01 NIMA $\leq$ median & $2.74(0.61-12.44)$ & $5.99(0.51-70.90)$ & $0.83(0.13-5.14)$ \\
\hline DQB1*03:01 NIMA > median & $5.77(1.21-27.52)$ & $6.65(0.61-72.76)$ & $4.40(0.91-21.30)$ \\
\hline ar category increase & $2.40(1.10-5.24)$ & $2.64(0.81-8.68)$ & $2.24(1.01-4.97)$ \\
\hline DQB1*06:02/03 NIMA, undetected & Ref. & Ref. & Ref. \\
\hline DQB1*06:02/03 NIMA $\leq$ median & $1.36(0.52-3.62)$ & $1.64(0.56-4.79)$ & $1.20(0.41-3.53)$ \\
\hline DQB1*06:02/03 NIMA > median & $0.51(0.14-1.83)$ & $0.50(0.13-1.92)$ & $1.31(0.46-3.77)$ \\
\hline Per category increase & $0.80(0.45-1.40)$ & $0.82(0.45-1.48)$ & $1.17(0.70-1.97)$ \\
\hline
\end{tabular}

aOR: adjusted OR; Cl: Confidence Interval.

Main analysis is shown leftmost for comparison, followed by an analysis including more covariates, and an analysis including the measured values of samples which were positive in only one of ten replicates (originally set to zero in the main analysis).

* adjusted for maternal age and pre-pregnancy BMI

$\dagger$ adjusted for maternal age, pre-pregnancy BMI, sustained smoking during pregnancy, caesarean section, childs' gender and HLA risk for T1D.

‡ combining DQB1*03:01, ${ }^{*} 04: 02$ and ${ }^{*} 06: 02 / 03$. The DQB1*04:02 NIMA subgroup was not analysed separately due to low number of children with measurable quantities in this group.

$\S$ the median value was calculated from controls with detectable levels. 
Figure 1: Study overview
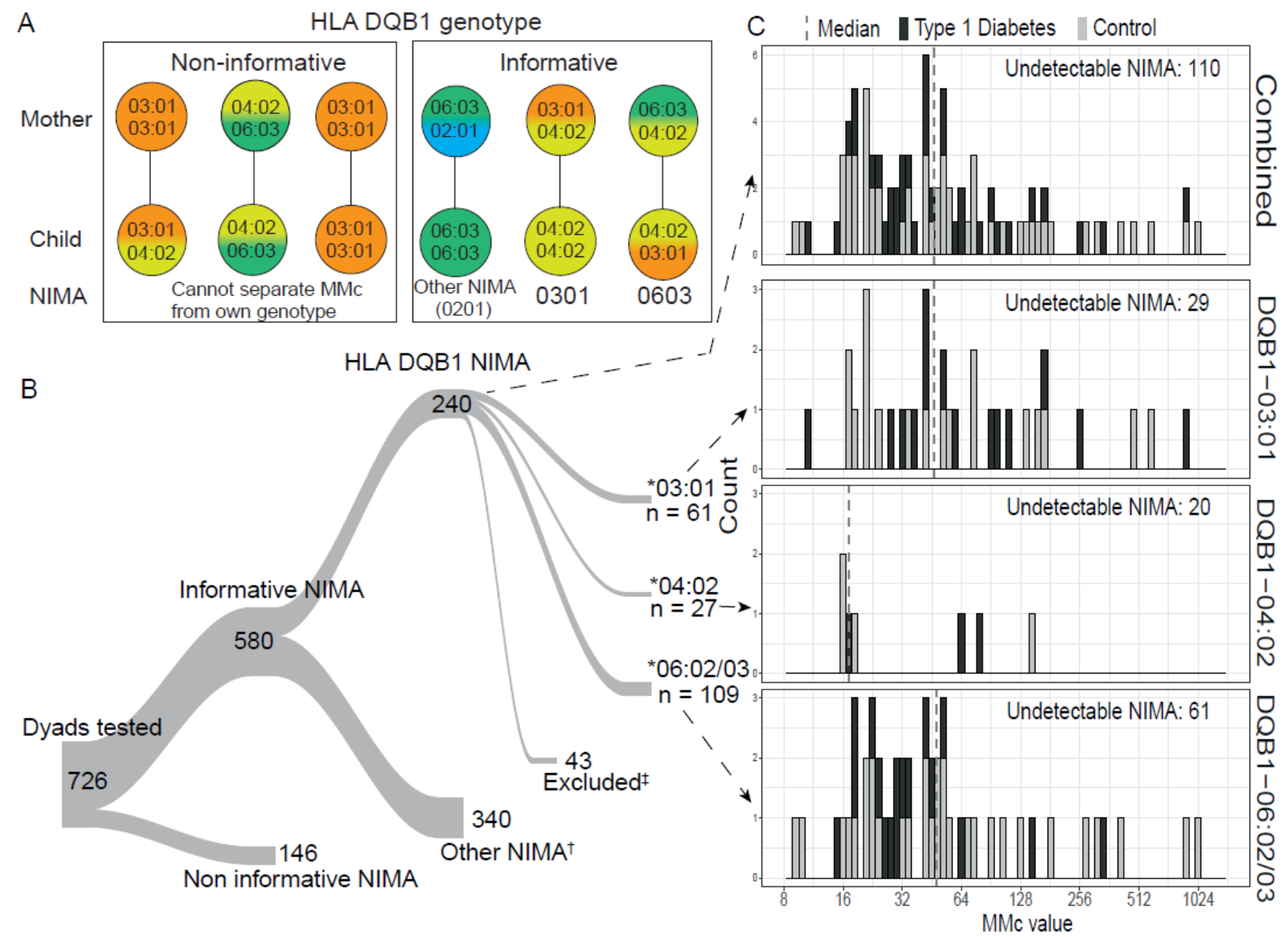

$\dagger$ Includes also dyads with a non-DQB1 NIMA $(n=42)$.

¥ This group includes samples $(n=26)$ that were tested with an earlier version of the ddPCR DQB1*03:01 probe which turned out to interact with other alleles, and were excluded. It also includes samples not found or with insufficient DNA $(n=8)$, samples with genomic levels of MMc $(n=8)$ potentially caused by contamination from maternal blood and maternal samples included by mistake $(n=1)$.

Figure 1 shows how the study design and distribution of participants: Panel A shows example HLA-DQB1 genotypes in mothers and offspring to illustrate whether a NIMA can be measured (informative NIMA), and the resulting NIMA used to measure MMc. Note that as HLA-DQB1*06:02 and *06:03 assay are considered one genotype in this study due to the assay used. NIMA was ascertained by comparing maternal and offspring genotypes. If the mother is homozygous, or if mother and child share both alleles, we are unable to measure MMc as we cannot determine NIMA and child's genomic levels of the genotype would mask the maternal signal (non-informative $M M c$ ). If the NIMA is informative (child has a NIMA that would not be masked by its own genotype), only the most common NIMA with ddPCR assays available were 
tested: HLA-DQB1*03:01, *04:02 or *06:02/03; others are referred to as "other NIMA". Panel B shows a Sankey diagram to illustrate how many dyads fall into each category in our cohort. Panel $C$ shows a histogram of observed MMc values in cord blood (in a log2 scale), in cases and controls, with the median of the positive samples (calculated using values from controls only) marked with a dashed line, and number of negative values listed in the top right corner of each facet. The top facet shows the total of all measured MMc, while the lower panels show per HLA genotype (HLADQB1*03:01, *04:02 and *06:02/03, in descending order). 